\title{
ANTITUMOR EFFECT OF KAZUSAMYCIN B ON EXPERIMENTAL TUMORS
}

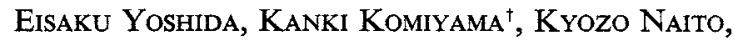 \\ Yoshinori Watanabe, Keiko Takamiya, Akira OKura, \\ Kohtarou Funaishi, Kenji KaWAMURA, \\ ShINJI Funayama ${ }^{\dagger}$ and IwaO UMEZaWA ${ }^{\dagger}$ \\ Exploratory Research Laboratories, Banyu Pharmaceutical Co., Ltd., \\ 2-9-3 Shimomeguro, Meguro-ku, Tokyo 153, Japan \\ tThe Kitasato Institute, \\ 5-9-1 Shirokane, Minato-ku, Tokyo 108, Japan
}

(Received for publication April 1, 1987)

\begin{abstract}
Kazusamycin B, a novel antibiotic (MW 542) isolated from fermentation broth of Streptomyces sp. No. 81-484 showed a broad antitumor spectrum both in vitro and in vivo. IC against the growth of tumor cells was around $1 \mathrm{ng} / \mathrm{ml}$ at 72 hours-exposure in vitro. Intraperitoneal injection of the antibiotic was effective in inhibiting the growth of murine tumors, S180, P388, EL-4, and B16. It was also active against doxorubicin-resistant P388, hepatic metastases of L5178Y-ML, pulmonary metastases of $3 \mathrm{LL}$, and human mammary cancer MX-1 xenografted to nude mice. However, the activity of kazusamycin B toward L1210 or human lung cancer LX-1 was weaker.

According to the results of comparative studies on the effect of kazusamycins B and A, an analog of $B$, there seemed to be no significant difference in their effectiveness. The effective dose range and toxicity were markedly dependent on tumor lines tested and the regimen used. Maximum tolerated dose in mice with subcutaneous tumors was much higher than that in mice bearing ascitic leukemia as P388. Although intermittent administration could greatly reduce the cumulative toxicity of the drug, therapeutic effect was similar with both successive and intermittent administration schedules.
\end{abstract}

In 1984, a highly lypophilic antitumor antibiotic named kazusamycin was obtained from the fermentation broth of Streptomyces sp. No. 81-484'). It was characterized by an unsaturated branchedchain aliphatic acid with a terminal $\delta$-lactone moiety ${ }^{2)}$. In the course of isolating kazusamycin from cultured broth, kazusamycin B (MW 542) was found as the major product with its congeners, kazusamycin A and leptomycins A and B. The fermentation, isolation, physico-chemical properties and chemical structure of kazusamycin B were reported recently ${ }^{32}$.

As presented previously ${ }^{4)}$, kazusamycin $\mathrm{A}$ has an unusual antitumor spectrum in vivo. Kazusamycin $\mathrm{A}$ is effective in inhibiting the development of spontaneous metastasis of B16 melanoma and Lewis lung carcinoma even at doses insufficient to suppress tumor growth at the implanted site ${ }^{5)}$. Antitumor activity of hydroxyelactocin (PD 114,721 structurally the same as kazusamycin A) was also extensively studied in addition to that of elactocin (CI-940) ${ }^{6}$.

In these studies the antitumor activity of kazusamycin A and its analog appeared to highly depend upon dosing regimen. Elactocin was reported to have slightly stronger antitumor activity than its counterpart hydroxyelactocin in a sensitive tumor model ${ }^{b)}$. Therefore, we decided to investigate the effect of kazusamycin B on various experimental tumors including human tumors with intermittent or daily administration regimens. The results are presented herein. 


\section{Materials and Methods}

Animals

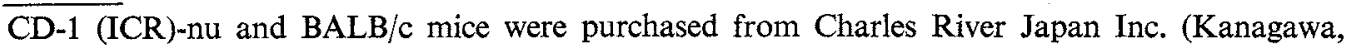
Japan). $\mathrm{BDF}_{1}(\mathrm{C} 57 \mathrm{BL} / 6 \times \mathrm{DBA} / 2), \mathrm{CDF}_{1}(\mathrm{BALB} / \mathrm{c} \times \mathrm{DBA} / 2)$, and $\mathrm{ICR}$ mice were from Shizuoka Laborotory Animal Center (Shizuoka, Japan). They were all specific pathogen free female. Except for nude mice, they were maintained in a barrier system, and were 6 or 7 weeks old at the start of each experiment. Nude mice were kept in an isolation box in the barrier and were 4 weeks old at the start of each experiment.

\section{Tumors}

Experiments were performed with 8 murine tumors, sarcoma 180 (S180), doxorubicin-resistant P388 leukemia (P388/ADR) and drug sensitive P388 (P388/S), L1210 leukemia, EL-4 lymphoid leukemia, L5178Y-ML lymphoma, B16 melanoma, and Lewis lung carcinoma (3LL), and 2 human tumors, LX-1 lung carcinoma and MX-1 mammary carcinoma. S180, P388, L1210, and EL-4 cells were maintained by weekly ip passage in ICR, DBA/2 (Charles River Japan Inc.), CDF 1 , C57BL/6 (Charles River Japan Inc.), respectively. P388/ADR cells were kindly given by Dr. TsuRuo of Japanese Foundation for Cancer Research. B16 and 3LL cells were maintained by biweekly subcutaneous passage of minced tumor nodules in C57BL/6 mice. Single-cell tumor suspensions were prepared by the usual mechanical method. The viability of tumor cells in both cases was approximately $60 \%$ based on trypan blue dye exclusion. L5178Y-ML, a variant of L5178Y lymphoma, is specifically matestatic to a liver. It was established by WATANaBe et al. of Banyu Pharmaceutical Co., Ltd. ${ }^{7)}$; it was selected by repeated intravenous passage of liver metastases and maintained by the same method. For experiments, liver tissue with metastasized L5178Y-ML cells was briefly dispersed and cultured in vitro to exclude hepatocytes for a week. Details of L5178Y-ML liver metastatic lymphoma will be soon reported elsewhere ${ }^{\text {7) }}$. LX-1 and MX-1 cells were gifts from Dr. INABA of Japanese Foundation for Cancer Research and kept by monthly subcutaneous passage in CD-1 (ICR)-nu mice.

For in vitro experiments, $\mathrm{L} 929, \mathrm{~B} 16$, and $\mathrm{HeLa} \mathrm{S} 3$ cells were maintained by successive culture in DULBECCO's modified minimum essential medium supplemented with $10 \%$ calf serum. L5178YML, P388/S and P388/ADR cells were cultured using RPMI medium 1640 supplemented with $10 \%$ fetal calf serum and $20 \mu \mathrm{M} \beta$-mercaptoethanol.

\section{Kazusamycins and Other Agents}

Kazusamycins $\mathrm{A}$ and $\mathrm{B}$ were prepared according to the method described by FUNAISHI et $a ._{0}{ }^{3)}$. For in vivo experiments, they were dissolved in small amounts of dimethyl sulfoxide and Tween 80 , and diluted with saline to make up the final dosing preparation containing $5 \%$ dimethyl sulfoxide and $0.6 \%$ Tween 80 . Mitomycin $\mathrm{C}$ and fluorouracil (5FU) were purchased from Kyowa Hakko Kogyo Co., Ltd. (Tokyo, Japan). Doxorubicin and cisplatin were from Sigma Chemical Co. (St. Louis, Mo., U.S.A.) and Nippon Kayaku Co., Ltd. (Tokyo Japan), respectively.

\section{In Vitro Cytotoxicity Assays}

L1210, P388/S, P388/ADR, B16, HeLa S3, L5178Y-ML and L929 cells were exposed to various concentration of kazusamycin $\mathrm{B}$ at $37^{\circ} \mathrm{C}$ for 72 hours in a $\mathrm{CO}_{2}$ incubator. Cytotoxicity toward $\mathrm{L} 1210$, P388/S, P388/ADR and L5178Y-ML cells was determined by counting the cell number with a Coulter counter. Effect on B16, HeLa S3, and L929 cells was examined by means of dye-staining methods ${ }^{8)}$.

\section{Antitumor Activity}

Antitumor activity toward ascitic tumors, S180, L1210, or P388 was evaluated by determination of survival time of mice. Activity against subcutaneously implanted B16, 3LL, MX-1 or LX-1 into a side flank of mice was investigated by measuring tumor weight. Pulmonary metastasis of $3 \mathrm{LL}$ was assessed by the increase of wet weight of lungs. In the case of EL-4 subcutaneous tumor, tumor size and survial period were measured instead of tumor weight. Effect on hepatic metastasis of L5178YML, which was generated by iv injection of L5178Y-ML cells, was evaluated in terms of liver-weight increase. Individual experimental conditions such as the number of tumor cells implanted, doses of 
kazusamycin B, treatment schedules, mouse strain, or experimental period are noted in the legends of tables in Results.

Statistical Analysis

All data were analyzed by Mann-Whitney U-test.

\section{Results}

\section{In Vitro Cytotoxicity}

$\mathrm{IC}_{50}$ of kazusamycin B against L1210, P388/S, B16, HeLa S3, L5178Y-ML or L929 was 1.8, 2.0, $0.7,1.8,10.0$ and $1.0 \mathrm{ng} / \mathrm{ml}$ respectively when cells were cultured with the drug for 72 hours. The growth of P388/ADR was also inhibitied $50 \%$ at a concentration of $5.4 \mathrm{ng} / \mathrm{ml}$. The potent cytotoxic action of kazusamycin $B$ appeared at an exposure period longer than 24 hours; $\mathrm{IC}_{50}$ on 8 and 24 hours exposure were 120 and $2.1 \mathrm{ng} / \mathrm{ml}$ toward HeLa S3 cells.

\section{Antitumor Effect on S180}

As shown in Table 1, when kazusamycin A or B were given intermittently, both were effective in prolonging the life span at a wide dose range. Kazusamycin $\mathbf{B}$ seemed to be a little more effective than kazusamycin A in prolonging the life span. While in the case of daily administration, the effect of kazusamycins A and B was almost the same at a dose 0.016 or $0.032 \mathrm{mg} / \mathrm{kg} / \mathrm{day}$. As in case of kazusamycin $\mathrm{A}^{4}$, kazusamycin $\mathrm{B}$ was more toxic when administered on the daily dose schedule than when given on the intermittent schedule.

\section{Antitumor Effect on P388 Leukemia}

As shown in Table 2, effective dose range of kazusamycin B was markedly wide on both schedules. Antitumor effect of kazusamycin B on P388/ADR cells was examined. As shown in Table 3, both

Table 1. Antitumor effect of kazusamycins B and A on $\mathrm{S} 180^{\mathrm{a}}$.

\begin{tabular}{|c|c|c|c|c|c|c|c|}
\hline \multirow{3}{*}{$\begin{array}{l}\text { Treatment } \\
\text { schedule }\end{array}$} & \multirow{3}{*}{$\begin{array}{c}\text { Dose } \\
(\mathrm{mg} / \mathrm{kg} / \text { day })\end{array}$} & \multicolumn{3}{|c|}{ Kazusamycin B } & \multicolumn{3}{|c|}{ Kazusamycin A } \\
\hline & & \multicolumn{3}{|c|}{ Survival days ${ }^{b}$} & \multicolumn{3}{|c|}{ Survival days } \\
\hline & & Mean $\pm S D$ & $P^{\mathrm{c}}<$ & ILS $(\%)$ & Mean $\pm S D$ & $P<$ & $\operatorname{ILS}(\%)$ \\
\hline Control & 0 & $14.8 \pm 4.0$ & & 0 & & & \\
\hline \multirow[t]{4}{*}{ Intermittent } & 0.027 & $29.4 \pm 7.7$ & 0.01 & 98 & $20.0 \pm 2.0$ & 0.05 & 35 \\
\hline & 0.053 & $27.0 \pm 7.9(2)$ & 0.05 & 82 & $20.3 \pm 1.5(1)$ & NS & 37 \\
\hline & 0.107 & $37.8 \pm 20.7(2)$ & 0.05 & 155 & $24.5 \pm 5.3(1)$ & 0.05 & 66 \\
\hline & 0.213 & $32.3 \pm 17.4(2)$ & 0.05 & 119 & NT & & \\
\hline \multirow[t]{5}{*}{ Successive } & 0.008 & $\mathrm{NT}$ & & & $26.3 \pm 6.7(1)$ & 0.01 & 77 \\
\hline & 0.016 & $32.0 \pm 13.4$ & 0.01 & 116 & $37.2 \pm 17.3$ & 0.01 & 151 \\
\hline & 0.032 & $31.5 \pm 15.4(1)$ & 0.05 & 113 & $29.0 \pm 7.4(1)$ & 0.01 & 96 \\
\hline & 0.064 & $15.2 \pm 10.5$ & NS & 5 & NT & & \\
\hline & 0.128 & $16.6 \pm 19.5$ & NS & 12 & NT & & \\
\hline
\end{tabular}

a ICR mice (control $n=10$, test $n=5$ ) were ip injected with $1 \times 10^{6}$ S180 cells on day 0 , and given drugs ip intermittently (day 1, 3 and 5) or successively (day $1 \sim 5$ ). Mean survival day of mice given $1 \mathrm{mg}$ / $\mathrm{kg}$ /day mitomycin $\mathrm{C}$ intermittently or successively was $33.0 \pm 15.1$ or $35.6 \pm 14.6$ days ( 126 or $141 \%$ ), respectively.

b Survivors (day 60) were excluded from the calculation. Number in parenthesis indicate 60-day survivors.

c Mann-Whitney U-test; NS, $P>0.05$.

NT: Not tested. 
Table 2. Antitumor effect of kazusamycin B on doxorubicin-sensitive P388 leukemia ${ }^{a}$.

\begin{tabular}{|c|c|c|c|c|c|}
\hline \multirow{2}{*}{$\begin{array}{c}\text { Drug } \\
\text { (ip) }\end{array}$} & \multirow{2}{*}{$\begin{array}{l}\text { Treatment } \\
\text { schedule }\end{array}$} & \multirow{2}{*}{$\begin{array}{c}\text { Dose } \\
\text { (mg/kg/day) }\end{array}$} & \multicolumn{3}{|c|}{ Survival days } \\
\hline & & & Mean $\pm S D$ & $P^{\mathrm{b}}<$ & ILS $(\%)$ \\
\hline None & & & $11.1 \pm 0.5$ & & 0 \\
\hline \multirow[t]{6}{*}{ Kazusamycin B } & Intermittent & 0.1 & $15.0 \pm 2.0$ & 0.01 & 35 \\
\hline & & 0.2 & $17.7 \pm 0.5$ & 0.01 & 59 \\
\hline & & 0.4 & $18.2 \pm 1.5$ & 0.01 & 64 \\
\hline & & 0.8 & $18.2 \pm 1.0$ & 0.01 & 64 \\
\hline & & 1.6 & $5.7 \pm 8.5$ & 0.05 & -49 \\
\hline & & 3.2 & $2.0 \pm 0.0$ & 0.05 & -82 \\
\hline \multirow[t]{4}{*}{ Kazusamycin B } & Successive & 0.013 & $15.8 \pm 1.3$ & 0.01 & 42 \\
\hline & & 0.025 & $16.2 \pm 0.8$ & 0.01 & 46 \\
\hline & & 0.050 & $16.2 \pm 0.4$ & 0.01 & 46 \\
\hline & & 0.100 & $17.0 \pm 2.0$ & 0.01 & 53 \\
\hline \multirow[t]{2}{*}{ Mitomycin C } & Successive & 0.25 & $12.7 \pm 1.2$ & 0.01 & 14 \\
\hline & & 1.00 & $15.3 \pm 0.8$ & 0.01 & 38 \\
\hline \multirow[t]{2}{*}{ Doxorubicin } & Successive & 0.5 & $19.3 \pm 2.2$ & 0.01 & 74 \\
\hline & & 2.0 & $25.5 \pm 4.5$ & 0.01 & 130 \\
\hline \multirow[t]{2}{*}{$5 F U$} & Successive & 5 & $15.2 \pm 0.8$ & 0.01 & 37 \\
\hline & & 20 & $18.8 \pm 1.0$ & 0.01 & 69 \\
\hline \multirow[t]{2}{*}{ Cisplatin } & Successive & 0.25 & $10.7 \pm 0.5$ & NS & -4 \\
\hline & & 1.00 & $18.5 \pm 1.4$ & 0.01 & 66 \\
\hline
\end{tabular}

a $\mathrm{CDF}_{1}$ mice (control $n=12$, test $n=6$ ) were ip injected with $1 \times 10^{6} \mathrm{P} 388$ cells on day 0 and given drugs intermittently (day $1,4,7,10$ and 13 ) or successively (day $1 \sim 5$ ).

b Mann-Whitney U-test; NS, $P>0.05$.

Table 3. Antitumor effect of kazusamycin B on doxorubicin-resistant P388 leukemia ${ }^{2}$.

\begin{tabular}{lccccc}
\hline \multirow{2}{*}{$\begin{array}{c}\text { Drug } \\
\text { (ip) }\end{array}$} & \multirow{2}{*}{$\begin{array}{c}\text { Treatment } \\
\text { schedule }\end{array}$} & $\begin{array}{c}\text { Dose } \\
(\mathrm{mg} / \mathrm{kg} / \mathrm{day})\end{array}$ & & \multicolumn{3}{c}{ Survival days } \\
\cline { 5 - 6 } & & & $10.3 \pm 0.6$ & & 0 \\
None & & & $11.8 \pm 1.2$ & 0.01 & 15 \\
Kazusamycin B & Intermittent & & $12.2 \pm 1.3$ & 0.01 & 19 \\
& & 0.2 & $13.7 \pm 1.4$ & 0.01 & 33 \\
& & 0.4 & $14.0 \pm 0.0$ & 0.01 & 37 \\
& & 0.8 & $14.0 \pm 0.6$ & 0.01 & 37 \\
& & 1.6 & $2.5 \pm 0.5$ & 0.01 & -76 \\
Kazusamycin B & Successive & 0.013 & $13.2 \pm 0.8$ & 0.01 & 28 \\
& & 0.025 & $14.8 \pm 2.6$ & 0.01 & 45 \\
& & 0.050 & $14.2 \pm 1.2$ & 0.01 & 38 \\
Mitomycin C & Successive & 0.100 & $15.0 \pm 2.1$ & 0.01 & 46 \\
& & 0.25 & $13.6 \pm 0.5$ & 0.01 & 32 \\
Doxorubicin & Successive & 2 & $18.5 \pm 1.5$ & 0.01 & 80 \\
& & 1.00 & $12.3 \pm 0.5$ & 0.01 & 20 \\
5FU & Successive & 5 & $11.2 \pm 0.8$ & 0.05 & 14 \\
& & 5 & $14.3 \pm 0.8$ & 0.01 & 40 \\
Cisplatin & Successive & 0.25 & $17.5 \pm 0.8$ & 0.01 & 71 \\
& & 1.00 & $13.5 \pm 0.8$ & 0.01 & 32 \\
& & $20.0 \pm 0.6$ & 0.01 & 94 \\
\hline
\end{tabular}

a $\mathrm{CDF}_{1}$ mice (control $n=12$, test $n=6$ ) were ip injected with $1 \times 10^{6} \mathrm{P} 388 / \mathrm{ADR}$ cells on day 0 and given drugs intermittently (day $1,4,7,10$ and 13 ) or successively (day $1 \sim 5$ ).

b Mann-Whitney U-test. 
Table 4. Antitumor effect of kazusamycin B on L1210 leukemia ${ }^{2}$.

\begin{tabular}{lcccc}
\hline \multirow{2}{*}{$\begin{array}{c}\text { Drug } \\
\text { (ip) }\end{array}$} & $\begin{array}{c}\text { Dose } \\
\text { (mg/kg/day) }\end{array}$ & Mean \pm SD & $p^{\mathrm{c}}<$ & SLS (\%) \\
\cline { 3 - 5 } & & $7.2 \pm 0.7$ & & 0 \\
None & 0.0063 & $7.0 \pm 0.0$ & $\mathrm{NS}$ & -3 \\
Kazusamycin B & 0.0125 & $7.8 \pm 0.4$ & $\mathrm{NS}$ & 8 \\
& 0.025 & $9.3 \pm 1.0$ & 0.01 & 29 \\
& 0.050 & $9.7 \pm 0.5$ & 0.01 & 35 \\
& 0.1 & $9.3 \pm 0.5$ & 0.01 & 29 \\
& 0.2 & $8.2 \pm 1.2$ & $\mathrm{NS}$ & 14 \\
Mitomycin C & 0.25 & $8.3 \pm 1.0$ & 0.05 & 15 \\
& 1.00 & $10.3 \pm 1.0$ & 0.001 & 43 \\
Doxorubicin & 0.5 & $10.7 \pm 1.4$ & 0.01 & 49 \\
& 2.0 & $17.8 \pm 4.1(1)$ & 0.01 & 147 \\
Cisplatin & 0.25 & $8.0 \pm 0.6$ & 0.05 & 11 \\
& 1.00 & $10.0 \pm 1.4$ & 0.01 & 39 \\
\hline
\end{tabular}

a $\mathrm{CDF}_{1}$ mice (control $n=15$, test $n=6$ ) were ip injected with $1 \times 10^{5}$ L1210 cells on day 0 and given drugs once daily from day 1 through death or day 10.

b A survivor (day 30) was excluded from calculation. Number in parenthesis indicate 30-day survivor.

e Mann-Whitney U-test; NS, $P>0.05$.

daily and intermittent administration of this drug increased the life span of mice. Toward this resistant line doxorubicin was ineffective at $2 \mathrm{mg} / \mathrm{kg}$ or even at a sub-lethal dose $5 \mathrm{mg} / \mathrm{kg}$.

Antitumor Effect on L1210 Leukemia

As shown in Table 4, kazusamycin B prolonged the survival period by 29 to $35 \%$ at a dose from 0.025 to $0.1 \mathrm{mg} / \mathrm{kg} /$ day.

Antitumor Effect on EL-4 Lymphoid Leukemia

As shown in Table 5, successive administration of kazusamycin B inhibited the tumor growth 57 or $94 \%$ at doses 0.1 or $0.2 \mathrm{mg} / \mathrm{kg} /$ day, respectively. The group administered $0.1 \mathrm{mg} / \mathrm{kg} / \mathrm{day}$ kazusamycin $\mathbf{B}$ had increased life span (ILS, not significant). Reference drugs, mitomycin C, doxorubicin, 5FU, and cisplatin, affected neither tumor growth nor the life span of mice.

\section{Antitumor Effect on B16 Melanoma}

Table 6 shows the results. When administered intermittently, at a dose $3.2 \mathrm{mg} / \mathrm{kg} / \mathrm{day}$, kazusamycin $\mathrm{B}$ inhibited the tumor growth $62 \%(P<0.01$ by $U$-test $)$. On a daily administration schedule, inhibition percent was 40 and $36 \%$ at doses 0.1 and $0.2 \mathrm{mg} / \mathrm{kg} /$ day. There was no significant difference between the effect of kazusamycin $\mathbf{B}$ and reference drugs entered. Antitumor effect of kazusamycin B was also tested on B16 inoculated into the peritoneal cavity of mice. When kazusamycin B was administered once daily from day 1 through day 5 , it prolonged the life span from 21.7 up to $26.8 \%$ at a dose 0.004 to $0.064 \mathrm{mg} / \mathrm{kg} /$ day. While mitomycin $\mathrm{C}$ prolonged the life span $27.4 \%$ at a dose $1 \mathrm{mg} / \mathrm{kg} /$ day.

\section{Antitumor Effect on $3 \mathrm{LL}$}

As shown in Table 7, the daily treatment with kazusamycin B at doses up to $0.2 \mathrm{mg} / \mathrm{kg} /$ day was ineffective against tumor growth at the implanted site, however, it inhibited pulmonary metastases by $59 \%$ in weight. Mitomycin $\mathrm{C}$ inhibited the primary tumor growth as well as the pulmonary metastases. 
Table 5. Antitumor effect of kazusamycin B on EL-4 lymphoid leukemia ${ }^{2}$.

\begin{tabular}{|c|c|c|c|c|c|c|}
\hline \multirow{2}{*}{$\begin{array}{l}\text { Drug } \\
\text { (ip) }\end{array}$} & \multirow{2}{*}{$\begin{array}{l}\text { Treatment } \\
\text { schedule }\end{array}$} & \multirow{2}{*}{$\begin{array}{c}\text { Dose } \\
\text { (mg/kg/day) }\end{array}$} & \multicolumn{2}{|c|}{ Tumor size $^{b}$} & \multicolumn{2}{|c|}{ Survival days } \\
\hline & & & $\mathrm{cm}^{2} \pm \mathrm{SD}$ & $\underset{(\%)}{\text { Inhibition }}$ & Mean $\pm S D$ & $\begin{array}{l}\text { ILS } \\
(\%)\end{array}$ \\
\hline None & & & $2.24 \pm 1.30$ & 0 & $20.9 \pm 7.3$ & 0 \\
\hline \multirow[t]{6}{*}{ Kazusamycin B } & Intermittent & 0.2 & $1.99 \pm 1.14$ & 11 & $19.1 \pm 1.1$ & -9 \\
\hline & & 0.4 & $2.42 \pm 1.20$ & -8 & $18.1 \pm 0.9$ & -13 \\
\hline & & 0.8 & $1.75 \pm 0.91$ & 22 & $20.1 \pm 2.2$ & -4 \\
\hline & & 1.6 & $1.34 \pm 0.91$ & 40 & $16.8 \pm 7.9$ & -20 \\
\hline & & 3.2 & $1.19 \pm 1.01$ & 47 & $13.0 \pm 7.9$ & -38 \\
\hline & & 6.4 & - & - & $3.3 \pm 0.7$ & -84 \\
\hline \multirow[t]{5}{*}{ Kazusamycin B } & Successive & 0.025 & $1.58 \pm 1.15$ & 29 & $20.3 \pm 4.3$ & -3 \\
\hline & & 0.05 & $1.54 \pm 1.53$ & 31 & $21.0 \pm 5.5$ & 0 \\
\hline & & 0.10 & $0.97 \pm 1.19^{\circ}$ & 57 & $27.3 \pm 13.0$ & 30 \\
\hline & & 0.20 & $0.14 \pm 0.28^{\mathrm{c}}$ & 94 & $21.6 \pm 18.4$ & 4 \\
\hline & & 0.40 & $0.90 \pm 1.27$ & 60 & $8.0 \pm 4.9$ & -62 \\
\hline \multirow[t]{2}{*}{ Mitomycin C } & Successive & 0.25 & $1.39 \pm 0.66$ & 38 & $20.5 \pm 3.1$ & -2 \\
\hline & & 1.00 & $1.82 \pm 0.59$ & 19 & $18.6 \pm 1.3$ & -11 \\
\hline \multirow[t]{2}{*}{ Doxorubicin } & Successive & 0.5 & $1.92 \pm 0.71$ & 14 & $18.8 \pm 1.7$ & -10 \\
\hline & & 2.0 & $1.23 \pm 1.16$ & 46 & $21.4 \pm 5.0$ & 2 \\
\hline \multirow[t]{2}{*}{$5 F U$} & Successive & 5 & $1.82 \pm 1.03$ & 19 & $19.8 \pm 5.3$ & -5 \\
\hline & & 20 & $1.64 \pm 0.78$ & 27 & $18.7 \pm 1.1$ & -11 \\
\hline \multirow[t]{2}{*}{ Cisplatin } & Successive & 0.25 & $1.40 \pm 1.30$ & 38 & $21.3 \pm 6.4$ & 2 \\
\hline & & 1.00 & $1.62 \pm 1.11$ & 28 & $19.0 \pm 0.9$ & -9 \\
\hline
\end{tabular}

a $\mathrm{BDF}_{1}$ mice (control $n=18$, test $n=8$ ) were subcutaneously injected with $3 \times 10^{5} \mathrm{EL}-4$ cells on day 0 and given drugs intermittently (day 1, 4, 7, 10 and 13) or successively (day $1 \sim 5$ ).

b Tumor size was measured on day 14 .

c $P<0.05$ by Mann-Whitney U-test.

-: Tumor size could not be measured because mice had died until day 5 .

Table 6. Antitumor effect of kazusamycin B on B16 melanoma ${ }^{\text {a }}$.

\begin{tabular}{|c|c|c|c|c|c|c|}
\hline \multirow{2}{*}{$\begin{array}{c}\text { Drug } \\
\text { (ip) }\end{array}$} & \multirow{2}{*}{$\begin{array}{l}\text { Treatment } \\
\text { schedule }\end{array}$} & \multirow{2}{*}{$\begin{array}{c}\text { Dose } \\
(\mathrm{mg} / \mathrm{kg} / \text { day })\end{array}$} & \multicolumn{3}{|c|}{ Tumor weight (g) } & \multirow{2}{*}{$\begin{array}{l}\text { Complete } \\
\text { regression }\end{array}$} \\
\hline & & & Mean $\pm S D$ & $P^{\mathrm{b}}<$ & $\begin{array}{c}\text { Inhibition } \\
(\%)\end{array}$ & \\
\hline None & & & $5.00 \pm 2.05$ & & 0 & \\
\hline \multirow[t]{5}{*}{ Kazusamycin B } & Intermittent & 0.2 & $4.02 \pm 2.14$ & NS & 20 & \\
\hline & & 0.4 & $4.82 \pm 2.46$ & NS & 4 & \\
\hline & & 0.8 & $4.09 \pm 2.11$ & NS & 18 & \\
\hline & & 1.6 & $3.94 \pm 1.31$ & NS & 21 & \\
\hline & & 3.2 & $1.92 \pm 1.08$ & 0.01 & 62 & 1 \\
\hline \multirow[t]{4}{*}{ Kazusamycin B } & Successive & 0.025 & $3.99 \pm 2.69$ & NS & 20 & \\
\hline & & 0.05 & $5.40 \pm 2.57$ & NS & -8 & \\
\hline & & 0.1 & $2.99 \pm 2.02$ & 0.05 & 40 & 1 \\
\hline & & 0.2 & $3.21 \pm 2.04$ & NS & 36 & \\
\hline \multirow[t]{2}{*}{ Mitomycin C } & Successive & 0.25 & $2.93 \div 1.12$ & 0.05 & 41 & \\
\hline & & 1.00 & $2.20 \pm 0.90$ & 0.01 & 56 & \\
\hline \multirow[t]{2}{*}{ Doxorubicin } & Successive & 0.5 & $3.00 \pm 2.34$ & NS & 40 & 1 \\
\hline & & 2.0 & $2.78 \pm 0.81$ & 0.01 & 56 & \\
\hline \multirow[t]{2}{*}{$5 \mathrm{FU}$} & Successive & 5 & $4.91 \pm 2.61$ & NS & 1 & \\
\hline & & 20 & $3.19 \pm 1.98$ & NS & 36 & \\
\hline \multirow[t]{2}{*}{ Cisplatin } & Successive & 0.25 & $4.37 \pm 1.84$ & NS & 14 & \\
\hline & & 1.00 & $4.35 \pm 1.54$ & NS & 13 & \\
\hline
\end{tabular}

a $\mathrm{BDF}_{1}$ mice (control $n=17$, test $n=8$ ) were subcutaneously injected with $1 \times 10^{6} \mathrm{~B} 16$ cells on day 0 and given drugs intermittently (day 1, 4, 7, 10 and 13) or successively (day $1 \sim 5$ ). Evaluation was done on day 22.

b Mann-Whitney U-test; NS, $P>0.05$. 
Table 7. Antitumor effect of kazusamycin B on the growth of primary tumors and pulmonary metastases of $3 \mathrm{LL}^{\mathrm{a}}$.

\begin{tabular}{|c|c|c|c|c|c|c|c|}
\hline \multirow{2}{*}{$\begin{array}{l}\text { Drug } \\
\text { (ip) }\end{array}$} & \multirow{2}{*}{$\begin{array}{c}\text { Dose } \\
(\mathrm{mg} / \mathrm{kg} / \mathrm{day})\end{array}$} & \multicolumn{3}{|c|}{ Primary tumor weight $(\mathrm{g})$} & \multicolumn{3}{|c|}{ Pulmonary weight (g) } \\
\hline & & Mean $\pm S D$ & $P^{\mathrm{b}}<$ & $\begin{array}{c}\text { Inhibition } \\
(\%)^{c}\end{array}$ & Mean $\pm S D$ & $P^{\mathrm{b}}<$ & $\begin{array}{l}\text { Inhibition } \\
(\%)^{c}\end{array}$ \\
\hline \multicolumn{2}{|c|}{ None (normal mice) } & & & & $0.130 \pm 0.013$ & & \\
\hline \multicolumn{2}{|c|}{ None (tumor bearers) } & $8.57 \pm 1.65$ & & 0 & $0.333 \pm 0.111$ & & 0 \\
\hline \multirow[t]{3}{*}{ Kazusamycin B } & 0.0125 & $9.58 \pm 1.70$ & NS & -12 & $0.320 \pm 0.102$ & NS & 6 \\
\hline & 0.05 & $7.19 \pm 1.31$ & NS & 16 & $0.344 \pm 0.131$ & NS & -5 \\
\hline & 0.20 & $6.90 \pm 2.07$ & NS & 19 & $0.213 \pm 0.049$ & 0.05 & 59 \\
\hline Mitomycin $\mathrm{C}$ & 1.00 & $5.81 \pm 0.74$ & 0.01 & 32 & $0.190 \pm 0.047$ & 0.05 & 70 \\
\hline
\end{tabular}

a $\mathrm{BDF}_{1}$ mice (control $n=16$, test $n=8$ ) were subcutaneously implanted with $5 \times 10^{5} 3 \mathrm{LL}$ cells on day 0 and given drugs once daily from day 1 to 5 . Evaluation was done on day 21 .

b Mann-Whitney U-test; NS, $P>0.05$.

c Inhibition of pulmonary weight increase due to metastases was calculated by the formula; $(\mathrm{A}-\mathrm{B}) /(\mathrm{A}-\mathrm{C})$ $\times 100(\%)$, A; lung weight of tumor bearing control group, B; lung weight of test group, C; lung weight of normal group.

Table 8. Antitumor effect of kazusamycin B on L5178Y-ML liver metastatic lymphoma ${ }^{a}$.

\begin{tabular}{|c|c|c|c|c|c|c|}
\hline \multirow{2}{*}{$\begin{array}{l}\text { Treatment } \\
\text { schedule } \\
\text { (ip) }\end{array}$} & \multirow{2}{*}{$\underset{\text { (mg/kg/day) }}{\text { Dose }}$} & \multicolumn{4}{|c|}{ Liver weight $(\mathrm{g}) \pm \mathrm{SD}$} & \multirow{2}{*}{$\begin{array}{l}\text { Inhibition }^{c} \\
\qquad \%)\end{array}$} \\
\hline & & With tumors & $P^{b}<$ & Without tumors & $P^{\mathrm{b}}<$ & \\
\hline & 0 & $2.58 \pm 0.30$ & & $0.86 \pm 0.05$ & & 0 \\
\hline Intermittent & 3.2 & $1.81 \pm 0.50$ & 0.01 & $1.05 \pm 0.07$ & 0.05 & 56 \\
\hline (day 1,4 and 7 ) & 6.4 & $1.19 \pm 0.30$ & 0.01 & $1.02 \pm 0.07$ & 0.05 & 90 \\
\hline Successive & 0.2 & $1.36 \pm 0.32$ & 0.01 & $0.94 \pm 0.19$ & NS & 78 \\
\hline (day $1 \sim 5$ ) & 0.4 & $1.01 \pm 0.39$ & 0.01 & $0.87 \pm 0.08$ & NS & 93 \\
\hline
\end{tabular}

a $\mathrm{CDF}_{1}$ mice (control $n=10$, test $n=8$ ) were injected with $4 \times 10^{5}$ L5178Y-ML cells into their tail vein on day 0 and measured their liver weight on day 8.

b Mann-Whitney U-test; NS, $P>0.05$.

c Inhibition $(\%)=(\mathrm{C}-\mathrm{T}) / \mathrm{C} \times 100$ where " $\mathrm{C}$ " is the liver weight increase in a control group $(2.58-0.86)$ and " $T$ " is the liver weight increase in each test group.

Table 9. Antitumor effect of kazusamycin B on human tumors MX-1 and LX-1 ${ }^{\text {a }}$.

\begin{tabular}{|c|c|c|c|c|}
\hline \multirow{2}{*}{ Tumors } & \multirow{2}{*}{$\begin{array}{c}\text { Dose } \\
(\mathrm{mg} / \mathrm{kg} / \text { day })\end{array}$} & Tumor weight (g) & \multirow{2}{*}{$\begin{array}{c}\text { Inhibition } \\
(\%)\end{array}$} & \multirow{2}{*}{$\begin{array}{c}\text { Body weight } \\
\text { change } \\
\text { (g) }\end{array}$} \\
\hline & & Mean $\pm S D$ & & \\
\hline \multirow[t]{3}{*}{ MX-1 } & 0 & $2.00 \pm 0.53$ & 0 & $+3.5(27.8-24.3)$ \\
\hline & 1.6 & $1.24 \pm 0.82$ & 38 & $+5.3(29.2-23.9)$ \\
\hline & 3.2 & $0.92 \pm 0.88$ & 54 & $+3.5(26.5-23.0)$ \\
\hline \multirow[t]{3}{*}{ LX-1 } & 0 & $2.26 \pm 1.60$ & 0 & $-1.1(19.1-20.2)$ \\
\hline & 1.6 & $1.41 \pm 0.63$ & 38 & $-1.3(19.8-21.1)$ \\
\hline & 3.2 & $2.41 \pm 0.50$ & -6 & $-2.0(18.8-20.8)$ \\
\hline
\end{tabular}

a CD-1 nude mice $(n=7)$ were inoculated subcutaneously with approximately $1 \mathrm{~mm}^{3}$ of non-necrotic nodule of MX-1 or LX-1 tumor. When the tumors developed to $100 \mathrm{~mm}^{3}$ in average (day $0: 18$ or 13 days after MX-1 or LX-1 implantation, respectively) mice were randomized and treated intravenously once a week for 5 times from day 0 . Tumors were weighed on day 35. The tumor weight of mice treated with $3 \mathrm{mg}$ of mitomycin $\mathrm{C} / \mathrm{kg}$ was $0 \mathrm{~g}$ (MX-1, $100 \%$ inhibition), or $0.53+0.48 \mathrm{~g}$ ( $\mathrm{LX}-1,76 \%$ inhibition).

$\mathrm{b}$ Difference of body weight on day 35 and day 0 , which includes tumor weight. Numbers in parenthesis indicate whole body weight after (left) and before (right) the treatment. 


\section{Antitumor Effect on the Liver Metastasis of L5178Y-ML Lymphoma}

As shown in Table 8, successive administration of kazusamycin B inhibited the experimental metastasis at doses 0.2 and $0.4 \mathrm{mg} / \mathrm{kg} /$ day. Intermittent administration was also effective at higher doses, 3.2 and $6.4 \mathrm{mg} / \mathrm{kg} /$ day. However, at those higher doses kazusamycin $\mathrm{B}$ induced a liver weight increase in reference mice without tumors.

\section{Antitumor Effect on Human Tumors}

As Table 9 shows, kazusamycin B inhibited the growth of MX-1 38 and $54 \%$ at doses 1.6 and $3.2 \mathrm{mg} / \mathrm{kg} / \mathrm{day}$, respectively. As for LX-1, the effect of kazusamycin B was equivocal, and body weight loss, which may due to cachexia(?) was observed in every mice implanted with the tumors.

\section{Discussion}

The present study was carried out 1) to compare the effect of kazusamycin B with that of A, 2) to evaluate the antitumor effect of kazusamycin $B$ on various experimental tumors including human tumor xenografts, and 3) to search for a proper regimen. As described above, kazusamycin B showed a broad antitumor spectrum similar to kazusamycin $A^{4)}$. It appeared that there was no significant difference in the antitumor activity between kazusamycins B and A. In addition, both antibiotics showed similar toxicity toward mice. $\mathrm{LD}_{50}$ of kazusamycins $\mathrm{A}$ and $\mathrm{B}$ in healthy female ICR mice were 7.0 and $9.4 \mathrm{mg} / \mathrm{kg}$, respectively. At autopsy, intraintestinal bleeding was observed in every dead mouse of both treatment groups. Kazusamycin A showed evidence of enterohepatic circulation ${ }^{9}$ including intraintestinal bleeding. Our observation suggests that the body distribution of kazusamycin B in mice is also similar to that of kazusamycin A. And it can explain the in vivo effect of kazusamycin B on L5178Y-ML in spite of its lower sensitivity to the antibiotic than the other cells lines. The antitumor effect of kazusamycin B on murine tumors such as S180, EL-4 or B16 was almost equal to clinically well used antitumor drugs. In some cases, the antitumor effect of kazusamycin B was superior to these drugs, whereas it was less effective in inhibiting the growth of P388/S and L1210 than doxorubicin.

Strong cytotoxic activity of kazusamycin B will explain its in vivo antitumor effect. On the other hand, we also suppose that antitumor immunity of tumor-bearing mice might play a role in the action of kazusamycin B as well as in the case of other known antitumor drugs ${ }^{10 \sim 15)}$. It is well known that S180 and B16 cells are highly antigenic. EL-4 is also antigenic when BDF ${ }_{1}$ mice were used as hosts ${ }^{8}$. Weak antitumor effect of kazusamycin B on human LX-1 and MX-1 xenografts might be also in part due to immune deficiency in nude mice, in addition to possible prospect such as these tumors were insensitive to kazusamycin B, or the treatment schedule was inadequate. Furthermore, as reported in this and a previous paper ${ }^{5)}$, kazusamycins $\mathrm{A}$ and $\mathrm{B}$ were effective in inhibiting spontaneous metastasis of $3 \mathrm{LL}$ at doses allowing the progression of primary tumors. It is a general understanding that the immune system participates in the development of metastasis to a certain extent, as well as that chemotherapeutic drugs may easily show an inhibitory effect on metastasis because of the smaller cell populations of metastasized foci than primary tumors, and that drug distribution may also be involved. Moreover, concerning kazusamycin A, its suppressing activity on bone marrow and splenocytes was weak $^{\text {(). }}$.

As for an effective regimen, both intermittent and successive administrations of kazusamycin B were equally effective. But effective dose range of each regimen was quite different. Time-dependent cytotoxicity of kazusamycin B will explain the difference.

\section{References}

1) Umezawa, I.; K. Komiyama, H. Oka, K. Okada, S. Tomisaka, T. Miyano \& S. Takano: A new antitumor antibiotic, kazusamycin. J. Antibiotics 37: 706 711, 1984

2) Komiyama, K.; K. OKada, H. OKa, S. Tomisaka, T. Miyano, S. Funayama \& I. Umezawa: Structural 
study of a new antitumor antibiotic, kazusamycin. J. Antibiotics 38:220 223, 1985

3) Funaishi, K.; K. Kawamura, Y. Sugiura, N. Nakahori, E. Yoshida, M. Okanishi, I. Umezawa, S. Funayama \& K. Komiyama: Kazusamycin B, a novel antitumor antibiotic. J. Antibiotics 40: 778 785,1987

4) Komiyama, K.; K. Okada, Y. Hirokawa, K. Masuda, S. Tomisaka \& I. Umezawa: Antitumor activity of a new antibiotic, kazusamycin. J. Antibiotics 38:224 229, 1985

5) Yoshida, E.; Y. Nishimuta, K. Natto, Y. Watanabe, S. Tomisaka, A. Okura, K. Komiyama \& I. UmeZAWA: The effect of kazusamycin on the growth of murine solid tumors and their spontaneous metastasis. J. Antibiotics 40: 391 393, 1987

6) Robert, B. J.; K. L. HAMelehle, J. S. Sebolt \& W. R. Leopold: In vivo and in vitro anticancer activity of the structurally novel and highly potent antibiotic CI-940 and its hydroxy analog (PD114, 721). Cancer Chemother. Pharmacol. 16: 95 101, 1986

7) Watanabe, Y.; A. Okura, K. Narto \& M. Kobayashr: Liver metastasis model using L5178Y-ML lymphoma and the effect of antitumor agents on the metastasis. J. Natl. Cancer Inst. in preparation

8) Okura, A.; K. Naito, H. Irzuka, M. Ishizuka, T. Takeuchi \& H. Umezawa: Effect of forphenicinol, a low molecular weight immunomodifier, on the growth of and the immune responses to murine lymphoma EL4. J. Biol. Resp. Modif. 6: 35 43, 1987

9) Umezawa, I. \& K. Komiyama: Studies on the new antibiotic kazusamycin and related substances. Jpn. J. Cancer Chemother. 14: 858 864, 1987

10) Hosokawa, M.: Modification of suppressor activity and effector activity of tumor-bearing hosts by bleomycin. Jpn. J. Cancer Chemother. 13:677 683, 1986

11) YAMAZAKI, M.: Tumor killing in cooperation with actinomycin D and neutrophils. Jpn. J. Cancer Chemother. 13: 684 688, 1986

12) Ogura, T.; H. Shindo, T. Masuno, S. Hosoe, S. Ikeda, S. Hayashi, I. Kawase \& S. Kishimoto: Induction of activated macrophages by intraperitoneal injection of mitomycin $\mathrm{C}$ in mice and rats. Jpn. J. Cancer Chemother. 13: 689 696, 1986

13) IshizukA, M.: Immunomodulation by antitumor antibiotics. Jpn. J. Cancer Chemother. 13: 697 700, 1986

14) KATAOKA, T.: Elimination of suppressor macrophages in tumor-bearing mice by 6-mercaptopurine and its therapeutic contribution. Jpn. J. Cancer Chemother. 13: 701 705, 1986

15) NitTa, K. \& T. TANAKA: Enhancement of immune response by an antitumor agent, cyclophosphamide. Jpn. J. Cancer Chemother. 13: 706 713, 1986 